\title{
Fenomenología depresiva al inicio de enfermedades neuropediátricas
}

\author{
Juan Narbona
}

Introducción. La depresión, en sus diversas formas, afecta al 8-10\% de niños y adolescentes y en la mayor parte de casos su origen es primario, siguiendo el modelo genético multifactorial. Pero hay una proporción de pacientes, no bien cuantificada todavía, en la que la depresión acompaña precozmente una enfermedad neurológica o marca un punto de inflexión en el curso de ésta. El objetivo es revisar la bibliografía al respecto.

Desarrollo. Se observa fenomenología depresiva, con una frecuencia significativamente mayor que por azar, en niños y adolescentes afectos de epilepsia, trastornos del sueño, cefaleas primarias crónicas recurrentes, enfermedades neurometabólicas y tumores intracraneales. En varias de estas patologías neuropediátricas se hipotetizan puntos de coincidencia fisiopatológica con la depresión a través de un déficit de disponibilidad cerebral de serotonina y noradrenalina. No se considera aquí la depresión disadaptativa a una neurodiscapacidad crónica.

Conclusiones. En niños y adolescentes, los trastornos del ánimo sintomáticos de enfermedad neurológica deben sospecharse en ausencia de antecedentes familiares o de experiencias vitales que los expliquen. La búsqueda sistemática de anomalías neurológicas y los exámenes complementarios permitirían en estos casos abordar precozmente el tratamiento de la enfermedad cerebral causante del cuadro depresivo. A su vez, como ocurre en casos de epilepsia, cefaleas o trastorno del sueño, la terapia farmacológica y psicológica del cuadro depresivo contribuye a mejorar la calidad de vida de los afectados.

Palabras clave. Cefalea crónica primaria. Depresión infanto-juvenil. Epilepsia. TDAH. Trastornos del sueño. Tumor cerebral.

\section{Introducción}

Los trastornos del ánimo, en sus diversas formas, afectan al 8-10\% de niños y adolescentes [1-8]. En una pequeña proporción de éstos, la sintomatología depresiva señala o forma parte del exordio de una enfermedad neurológica y, en ocasiones, marca una inflexión en su curso. El presente trabajo tiene como objetivo revisar la bibliografía relevante al respecto.

\section{Epilepsia y trastorno del ánimo en niños}

Varios trabajos han mostrado la presencia de sintomatología depresiva y ansiedad al inicio de una enfermedad epiléptica en niños [9]. En un estudio sobre 21 niños con epilepsia benigna rolándica de inicio reciente [10] se observó la correlación múltiple del índice de puntas interictales durante el electroencefalograma de vigilia y sueño con la gravedad de los síntomas depresivos $(p<0,001)$ y con los de agresividad y trastornos de conducta $(p<0,01)$. En otra investigación [11], mediante entrevista semiestructurada de 53 niños de 6-18 años con epilepsia primaria de comienzo reciente frente a 50 contro- les, se detectó fenomenología depresiva en el 22,6\% de pacientes y en el $4 \%$ de controles $(p<0,01)$; en bastantes casos la depresión se había notado antes de comenzar las primeras crisis, lo que lleva a suponer que ya en el estadio preclínico de la epilepsia había una disfunción de neurotransmisores con la actividad paroxística subclínica. Loney el al [12], utilizando el Children's Depression Inventory [13], hallaron que el $22 \%$ de los niños que iniciaban una epilepsia presentaban tristeza, anhedonia y descenso de logro escolar ( $p<0,05$ frente al grupo control), junto con déficit de atención y ansiedad. Otro trabajo prospectivo, utilizando la Child Behavior Checklist (CBCL) [14,15] en 242 niños que habían iniciado una epilepsia idiopática entre las edades de 4 y 14 años, mostraba una proporción significativamente mayor de síntomas internalizantes (depresión, ansiedad, miedos) frente al grupo control constituido por sus hermanos sanos.

Esta comorbilidad no azarosa entre epilepsia y depresión, independientemente de los efectos debidos a los fármacos $[9,16]$, ha generado estudios sistemáticos e investigaciones sobre las bases fisiopatológicas que ambos trastornos comparten $[9,17$ 20]. Mediante tomografía por emisión de positro-
Unidad de Neurología Pediátrica. Departamento de Pediatría. Clínica Universidad de Navarra. Pamplona, Navarra, España.

Correspondencia:

Dr. Juan Narbona García. Unidad de Neuropediatría. Clínica Universidad de Navarra. Avda. Pío XII, 36. E-31008 Pamplona (Navarra).

E-mail:

jnarbona@unav.es

Financiación:

Artículo elaborado con ayuda de la Fundación Fuentes Tudor (Pamplona) para investigación en neurología y neuropsicología del desarrollo.

Declaración de intereses:

El autor manifiesta la inexistencia de conflictos de interés en relación con este artículo.

Aceptado tras revisión externa: 10.01.14.

Cómo citar este artículo: Narbona J. Fenomenología depresiva al inicio de enfermedades neuropediátricas. Rev Neurol 2014; 58 (Supl 1): S71-5.

(c) 2014 Revista de Neurología 
nes se ha demostrado, en epilepsia temporal por esclerosis mesial, un defecto de serotonina y de noradrenalina en el hipocampo, la amígdala y la corteza cingulada anterior y orbitofrontal [21,22]. En animales de experimentación, la epileptogénesis inducida provoca conducta depresiva, y a la inversa, el estado depresivo inducido hace descender el dintel de epileptogénesis [23-25]. Se ha interpretado que el distrés debido a la actividad paroxística provoca, mediando la hipercortisolemia, un estado hiperglutamatérgico y un descenso de serotonina y de noradrenalina en el cerebro, que sustentarían la tendencia a la depresión y a la ocurrencia de crisis epilépticas [26,27].

\section{Disfunciones primarias del sueño y trastornos del ánimo en niños}

Se describe una relación multidireccional entre insuficiencia de sueño, trastornos del ánimo y trastorno de ansiedad [28]. Entre los primeros síntomas de la depresión mayor se describen sueño fragmentado e inquieto con despertar precoz y fatiga diurna. Los niños con trastorno depresivo subsindrómico y trastorno distímico autoperciben su sueño como no reparador, si bien los estudios polisomnográficos en estos casos no han confirmado las quejas subjetivas [29]. En niños y adolescentes con trastorno bipolar, el insomnio es un hecho clínico cardinal que puede estar en primer plano al comienzo del episodio [8].

De otra parte, la falta de sueño y la excesiva somnolencia diurna se acompañan frecuentemente de depresión subsindrómica, y ésta puede ser el motivo de consulta habitual. El síndrome de apnea obstructiva afecta al $1 \%$ de la población infantil y adolescente con motivo de obesidad, gran hipertrofia amigdalar, anomalías craneofaciales, mucopolisacaridosis y enfermedades neuromusculares [30-32]. Un 20\% de adolescentes con síndrome de Kleine-Levin padece depresión comórbida o secundaria a la misma disfunción cerebral episódica [33], y un 27\% de niños y adolescentes con síndrome de piernas inquietas (que prevalece un $2 \%$ en estas edades) cursa también con depresión subsindrómica o distimia [34].

\section{Cefaleas crónicas recurrentes y sintomatología depresiva}

Está bien documentada la coocurrencia de depresión en el curso de cefaleas crónicas idiopáticas en niños y adolescentes, aunque no sea tan clara esa asociación al inicio de la enfermedad cefalálgica. Un metaanálisis reciente [35] incluye diez publicaciones de series pediátricas en las que el tipo de cefalea estaba bien especificado, se incluía grupo de control y se había realizado a todos los sujetos un cribado de psicopatología con la CBCL [14]. Según este metaanálisis, los niños y adolescentes con cefalea crónica presentan una frecuencia más alta que los controles $(p<0,001)$ de síntomas internalizados (depresión, ansiedad), que no se muestran ligados a la frecuencia de episodios cefalálgicos ni al grado de discapacidad que ocasionan. En el estudio prospectivo de Pine et al [36] se siguieron 776 sujetos de 9-18 años a lo largo de nueve años, usando la entrevista semiestructurada Diagnostic Interview Schedule for Children [37], y se observó asociación a través del tiempo entre depresión y cefaleas; en el grupo de adolescentes depresivos, la prevalencia de episodios cefalálgicos era doble que en los no depresivos, y entre los afectos de depresión mayor, el riesgo de desarrollar cefalea crónica en los siguientes siete años era diez veces mayor que en los no depresivos. En una investigación [38] sobre 280 sujetos de ambos sexos de 4-14 años con cefaleas crónicas primarias, se encontró comorbilidad muy alta de cefalea crónica, depresión y ansiedad $(p<0,001$ frente a controles). Se especula que la depresión y la cefalalgia crónica tengan una fisiopatología compartida, a través de un déficit serotoninérgico cerebral [39-41].

\section{Afecciones neurometabólicas: enfermedad de Wilson y fenillcetonuria}

En las series más numerosas con degeneración hepatolenticular por déficit en ceruloplasmina (enfermedad de Wilson), un 2,5\% de pacientes inicia la clínica sólo con fenomenología psiquiátrica y un $16 \%$ adicional asocia síntomas de disfunción neuromotriz; en adolescentes o adultos jóvenes, los trastornos del ánimo (depresión y, en ocasiones, manía) son los cuadros psicopatológicos más frecuentes [42-45]. El cribado clínico y analítico de enfermedad de Wilson, como afección neurometabólica eficazmente tratable, debe formar parte de todo estudio inicial de un adolescente con trastorno del ánimo [46]; en todos los casos hay antecedentes de ictericia o marcadores bioquímicos actuales de afectación hepática. Algunos pacientes con enfermedad de Wilson tienen hipersensibilidad a neurolépticos, que se manifiesta por tormenta distónica o distónico-discinética [44]. Los síntomas depresivos son frecuentes también durante el curso de la enfermedad y así, en 
adultos con enfermedad de Wilson, la gravedad depresiva en la escala de Hammilton correlaciona con la biodisponibilidad de receptores sinápticos de serotonina -investigados mediante tomografía computariza por emisión de fotón único- en el hipotálamo, en el cerebro mesial y en la región superior del tronco cerebral, respecto al cerebelo [47].

En el informe original de la 'oligofrenia fenilpirúvica' (fenilcetonuria) de Følling [48], los niños afectos se describían tristes, enfadados, ansiosos, proclives a las rabietas, irritados, insociables y catatónicos, además de retrasados en su desarrollo. El tratamiento dietético temprano y continuado salva el desarrollo intelectual en estos pacientes, pero los síntomas de depresión, ansiedad y labilidad atencional son frecuentes, quizá por desequilibrios de catecolaminas, pero también ligados a la restricción en el estilo de vida [49-51].

\section{Tumores intracraneales y fenomenología depresiva inicial}

Una investigación sistemática [52] recuestionó la sintomatología presente durante los seis meses anteriores al diagnóstico de tumor intracraneal primario en 74 niños con edades de 0-16 años, mediante entrevistas a sus padres y aplicación de cuestionarios de cribado de psicopatología referida a la citada etapa prediagnóstica. Los síntomas más frecuentes eran cefaleas y vómitos (65\%), pero al mismo tiempo los padres habían notado cambios de comportamiento en el $52 \%$ de los pacientes: entre 0 y 5 años destacaban los trastornos del hábito de sueño, la irritabilidad y la inusual demanda de atención; los mayores de 5 años se manifestaban predominantemente con cambios de humor, tristeza, escasa concentración, conducta solitaria y ansiedad. En otro estudio [53], mediante la revisión de historias clínicas de 200 pacientes pediátricos con tumor primario intracraneal, se registró la presencia inicial de cambios de conducta en el 19\% de casos; estas alteraciones se describen como labilidad del humor, irritabilidad, agresividad y cambio del carácter. Estos dos estudios son los únicos que abordan con cierto detalle y sistemática el estado mental de los niños en el periodo precedente al diagnóstico del tumor intracraneal, que se demoró una media de seis meses tras el comienzo de los síntomas citados. Por el contrario, un metaanálisis [54] de 74 artículos publicados entre 1991 y 2005 que incluían suficiente información sobre la sintomatología inicial de 4.171 niños de 0-18 años afectos de tumores del sistema nervioso central, informaba de que sólo el
7\% mostraban alteraciones conductuales o disminución del rendimiento escolar; en el $4 \%$ de los menores de 4 años la irritabilidad era una manifestación inicial prominente. Debe prestarse suficiente importancia a los cambios de comportamiento y del humor en niños que padecen cefaleas o vómitos matinales, pero no ligados a rechazo a la escuela, sino a la hipertensión intracraneal y al sufrimiento de regiones centromesiales del cerebro, con probables alteraciones de neurotransmisores que podrían justificar la sintomatología temprana afectiva y comportamental.

En un metanálisis [55] de 21 adolescentes (siete varones) con anorexia nerviosa y caquexia, ocho sujetos (de ellos, cuatro varones) presentaban clínica depresiva predominante; la ausencia de aversión a la obesidad, la presencia de sintomatología atípica y los exámenes complementarios condujeron a la confirmación de un tumor basocerebral que afectaba, al menos, las paredes del hipotálamo.

\section{Comorbilidad de trastorno del ánimo, trastorno por déficit de atención/ hiperactividad y trastorno de coordinación}

El trastorno por déficit de atención/hiperactividad (TDAH), especialmente el subtipo con predominio de inatención, cuyo diagnóstico puede retrasarse más que en el subtipo combinado, se acompaña de trastorno comórbido del ánimo y de ansiedad en el $30-40 \%$ de casos, y éste puede ser el motivo inicial de consulta [56,57], junto con trastorno del sueño [58]. La forma de TDAH con predominio de inatención se acompaña muy frecuentemente -casi nueve de cada diez casos, en nuestra experiencia- $[59,60]$ de trastorno de la coordinación y de la comunicación social [4]. El trastorno bipolar puede ser manifiesto ya durante la niñez y especialmente en comorbilidad con TDAH de tipo combinado $[7,8]$.

\section{Conclusiones}

$\mathrm{Al}$ inicio de varias grandes patologías neuropediátricas puede observarse fenomenología depresiva. Los trastornos del ánimo se asocian inicialmente con especial frecuencia a epilepsias, trastornos del sueño y TDAH; menos frecuentemente acompañan el comienzo clínico de un proceso expansivo intracraneal o de una enfermedad neurometabólica. Es preciso prestar atención sistemáticamente a la fenomenología depresiva en el estudio clínico de pacientes neuropediátricos; $y$, a la inversa, pensar en 
enfermedad cerebral cuando no hay antecedentes familiares o eventos vitales que expliquen un trastorno del ánimo en edad infanto-juvenil. En referencia a las patologías neuropediátricas aquí estudiadas se hipotetizan puntos de coincidencia fisiopatológica con la depresión por un déficit de disponibilidad cerebral de serotonina o noradrenalina. Para el tratamiento de la depresión en niños y adolescentes se utilizan en la actualidad inhibidores selectivos de la recaptación de serotonina e intervenciones psicológicas que no sólo mejoran el estado de ánimo, sino también el curso de la afección neurológica y la calidad de vida del paciente. De otra parte, si la enfermedad neurológica mejora, también lo hará la sintomatología depresiva.

Bibliografía

1. Costello EJ, Mustillo S, Erkanli A, Keeler G, Angold A. Prevalence and development of psychiatric disorders in childhood and adolescence. Arch Gen Psychiatry 2003; 60: 837-44.

2. Fergusson DM, Horwood LI, Ridder EM, Beautrais AL. Subthreshold depression in adolescence and mental health outcomes in adulthood. Arch Gen Psychiatry 2005; 62: 66-72.

3. American Psychiatric Association. Diagnostic and statistical manual of mental disorders, fourth edition, text revised (DSM-IV-TR). Washington DC: APA; 2000.

4. American Psychiatric Association. Diagnostic and statistical manual of mental disorders, fifth edition (DSM-5). Washington DC: APA; 2013

5. Birmaher B, Ryan ND, Williamson DE, Brent DA, Kaufman J, Dahl RE, et al. Childhood and adolescent depression: a review of the past 10 years. Part I. J Am Acad Child Adolesc Psychiatry 1996; 35: 1427-39.

6. Lewinsohn PM, Klein, Seeley JR. Bipolar disorders in a community sample of older adolescents: prevalence, phenomenology, comorbidity, and course. J Am Acad Child Adolesc Psychiatry 1995; 34: 454-63.

7. Soutullo CA, Chang KD, Díez-Suárez A, Figueroa-Quintana A, Escamilla-Canales I, Rapado-Castro M. Bipolar disorders in children and adolescents: international perspective on epidemiology and phenomenology. Bipolar Disord 2005; 7: 497-506.

8. Soutullo CA, Escamilla I, Wozniak J, Gamazo P, Figueroa A, Biederman J. Phenomenology of pediatric bipolar disorder in a Spanish sample: features before and at the moment of the diagnosis. J Affect Disord 2009; 118: 39-47.

9. Reilly C, Agnew R, Neville BGR. Depression and anxiety in childhood epilepsy: a review. Seizure 2011; 20: 589-97.

10. Sarco DP, Boyer K, Lundy-Krigbaum SM, Masanori Takeoka M Jensen F, Gregas M, et al. Benign rolandic epileptiform discharges are associated with mood and behavior problems. Epilepsy Behav 2011; 22: 298-303.

11. Jones JE, Watson R, Sheth R, Caplan R, Koehn M, Seidenberg M, et al. Psychiatric comorbidity in children with new onset epilepsy. Dev Med Child Neurol 2007; 49: 493-7.

12. Loney JC, Wirrell EC, Sherman EMS, Hamiwka LD. Anxiety and depressive symptoms in children presenting with a first seizure. Pediatr Neurol 2008; 39: 236-40.

13. Kovacs M. Children's Depression Inventory: technical manual update. Toronto: Multi-Health Services; 2003.

14. Austin JK, Dunn DW, Caffrey HM, Perkins SM, Harezlak J, Rose DF. Recurrent seizures and behavior problems in children with first recognized seizures: a prospective study. Epilepsia 2002; 43: 1564-73.

15. Achenbach TM. Manual for Child Behavior Checklist/4-18 and 1991 profile. Burlington: University of Vermont; 1991.
16. Caplan R. Psychopathology in pediatric epilepsy: role of antiepileptic drugs. Front Neurol 2012; 3: 163.

17. Plioplys S. Depression in children and adolescents with epilepsy. Epilepsy Behav 2003; 4 (Suppl 3): S39-45.

18. Ekinci O, Titus JB, Rodopman AA, Berkem M, Trevathan E. Depression and anxiety in children and adolescents with epilepsy: prevalence, risk factors, and treatment. Epilepsy Behav 2009; 14: 8-18.

19. Kerr MP, Mensah S, Besag F, De Toffol B, Ettinger A, Kanemoto K, et al; International League Against Epilepsy (ILAE) Commission on the Neuropsychiatric Aspects of Epilepsy. International consensus clinical practice statements for the treatment of neuropsychiatric conditions associated with epilepsy. Epilepsia 2011; 52: 2133-8.

20. Kanner AM, Schachter SC, Barry JJ, Hersdorffer DC, Mula M, Trimble M, et al. Depression and epilepsy: epidemiologic and neurobiologic perspectives that may explain their high comorbid occurrence. Epilepsy Behav 2012; 24: 156-68.

21. Merlet I, Ostrowsky K, Costes N, Ryvlin P, Isnard J, Faillenot I, et al. 5-HT1A receptor binding and intracerebral activity in temporal lobe epilepsy: an [18F]MPPF-PET study. Brain 2004; 127: 900-13.

22. Salzberg M, Taher T, Davie M, Carne R, Hicks RJ, Cook M, et al. Depression in temporal lobe epilepsy surgery patients: an FDG-PET study. Epilepsia 2006; 47: 2125-30.

23. Victoroff JI, Benson F, Grafton ST, Engel J Jr, Mazziotta JC. Depression in complex partial seizures. Electroencephalography and cerebral metabolic correlates. Arch Neurol 1994; 51: 155-63.

24. Salzberg M, Kumar G, Supit L, Jones NC, Morris MJ, Rees S, et al. Early postnatal stress confers enduring vulnerability to limbic epileptogenesis. Epilepsia 2007; 48: 2079-85.

25. Gilby KL, Sydserff S, Patey AM, Thorne V, St-Onge V, Jans J, et al. Postnatal epigenetic influences on seizure susceptibility in seizure-prone versus seizure-resistant rat strains. Behav Neurosci 2009; 123: 337-46.

26. Zobel A, Wellmer J, Schulze-Rauschenbach S, Pfeiffer U, Schnell S, Elger C, et al. Impairment of inhibitory control of the hypothalamic pituitary adrenocortical system in epilepsy. Eur Arch Psychiatry Clin Neurosci 2004; 254: 303-11.

27. Mazarati AM, Shin D, Kwon YS, Bragin A, Pineda E, Tio D, et al. Elevated plasma corticosterone level and depressive behavior in experimental temporal lobe epilepsy. Neurobiol Dis 2009; 34: 457-61.

28. Chorney DB, Detweiler MF, Morris TL, Kuhn BR. The interplay of sleep disturbance, anxiety, and depression in children. J Pediatr Psychol 2008; 33: 339-48.

29. Bertocci MA, Dahl RE, Williamson DS, Iosif AM, Birmaher B, Axelson D, et al. Subjective sleep complaints in pediatric depression: a controlled study and comparison with EEG measures of sleep and waking. J Am Acad Child Adolesc Psychiatry 2005; 44: 1158-66.

30. Aronen ET, Liukkonen K, Simola P, Virkkula P, Uschakoff A, Korkman M, et al. Mood is associated with snoring in preschoolaged children. J Dev Behav Pediatr 2009; 30: 107-14.

31. Carotenuto M, Esposito M, Parisi L, Gallai B, Marotta R, Pascotto A, et al. Depressive symptoms and childhood sleep apnea syndrome. Neuropsychiatr Dis Treat 2012; 8: 369-73.

32. Yglesias A, Narbona J, Vanaclocha V, Artieda J. Chiari type I malformation, glossopharyngeal neuralgia and central sleep apnoea in a child. Dev Med Child Neurol 1996; 38: 1126-30.

33. Dauvilliers Y, Mayer G, Lecendreux M, Neidhart E, Peraita-Adrados R, Sonka K, et al. Kleine-Levin syndrome. An autoimmune hypothesis based on clinical and genetic analyses. Neurology 2002; 59: 1739-45.

34. Picchietti MA, Picchietti DL. Advances in pediatric restless legs syndrome: iron, genetics, diagnosis and treatment. Sleep Med 2010; 11: 643-51.

35. Balottin U, Poli PF, Termie C, Molteni S, Galli F. Psychopathological symptoms in child and adolescent migraine and tension-type headache: a meta-analysis. Cephalalgia 2012; 33: 112-22.

36. Pine DS, Cohen P, Brook J. The association between major depression and headache: results of a longitudinal 
epidemiologic study in youth. J Child Adolesc Psychopharmacol 1996; 6: 153-64.

37. Costello A. Report on the NIMH. Diagnostic Interview Schedule for Children (DISC). Research Forum Structured Diagnostic Instruments in Child Psychiatry. San Francisco, California; 1983.

38. Pavone P, Rizzo R, Conti I, Verrotti A, Mistretta A, Falsaperla R, et al. Primary headaches in children: clinical findings on the association with other conditions. Int J Immunopathol Pharmacol 2012; 5: 1083-91.

39. Ferrari M, Saxena P. On serotonin and migraine: a clinical and pharmacological review. Cephalalgia 1993; 13: 151-65.

40. Del Zompo M, Cherchi A, Palmas MA, Ponti M, Bocchetta A Gessa GL, et al. Association between dopamine receptor genes and migraine without aura in a Sardinian sample. Neurology 1998; 51: 781-6.

41. Silberstein SD. Shared mechanisms and comorbidities in neurologic and psychiatric disorders. Headache 2001; 41 (Suppl 1): S11-7.

42. Walshe JM. Wilson's disease. In Vinken PJ, Bruyn GW, Klawans HL, eds. Handbook of clinical neurology. Vol. 49. Amsterdam: Elsevier; 1986. p. 223-38.

43. Denning TR, Berrios GE. Wilson's disease: a longitudinal study of psychiatric symptoms. Biol Psychiatry 1990; 28: 255-65.

44. Taly AB, Meenakshi-Sundaram S, Sinha S, Swamy HS, Arunodaya GR. Wilson disease. Description of 282 patients evaluated over three decades. Medicine 2007; 82: 112-21.

45. Srinivas K, Sinha S, Taly AB, Prashanth LK, Arunodaya GR, Janardhana-Reddy YC, et al. Dominant psychiatric manifestations in Wilson's disease: a diagnostic and therapeutic challenge! J Neurol Sci 2008; 266: 104-8.

46. Woerwag-Mehta S, Hedderly P, Hedderly T, Dhawan A. Complex psychiatric presentation in adolescent onset Wilson's disease. BMJ Case Rep 2011; Jan 11. [Epub ahead of print].

47. Hessel S, Barthel H, Hermann W, Murai T, Kluge R, Wagner A, et al. Regional serotonin transporter availability and depression are correlated in Wilson's disease. J Neural Transm 2003; 110 923-33.

48. Følling A. Uber Ausscheidung von Phenylbrenztraubensaure in den Harn als Stoffwechselanomalie in Verbingdung mit Imbezillitat. Hoppe-Seylers Z Physiol Chem 1934; 227: 169-76.

49. Sullivan JE, Chang P. Review: emotional and behavioral functioning in phenylketonuria. J Pediatr Psychol 1999; 24: 281-99.

50. Brumm VL, Bilder D, Waisbren SE. Psychiatric symptoms and disorders in phenylketonuria. Mol Genet Metab 2010; 99 (Suppl): S59-63.

51. Weglage J, Grenzebach M, Pietsch M, Feldmann R, Linnenbank R, Denecke J, et al. Behavioural and emotional problems in early-treated adolescents with phenylketonuria in comparison with diabetic patients and healthy controls. J Inherit Metab Dis 2000; 23: 487-96.

52. Edgeworth J, Bullock P, Bailey A, Gallagher A, Crouchman M. Why are brain tumours still being missed? Arch Dis Child 1996; 74: 148-51.

53. Wilne SH, Ferris RC, Nathwani A, Kennedy CR. The presenting features of brain tumours: a review of 200 cases. Arch Dis Child 2006; 91: 502-6.

54. Wilne S, Collier J, Kennedy C, Koller K, Grundy R, Walker D. Presentation of childhood CNS tumours: a systematic review and meta-analysis. Lancet Oncol 2007; 8: 685-95.

55. Chipkevitch E. Brain tumors and anorexia nervosa syndrome. Brain Dev 1994; 16: 175-9.

56. Biederman J. Attention-deficit/hyperactivity disorder: a selective overview. Biol Psychiatry 2005; 57: 1215-20.

57. García-Giral M. Comorbilidad del trastorno por déficit de atención e hiperactividad con trastornos de ansiedad y con depresión mayor. In Soutullo-Esperón C, ed. Guía básica de psicofarmacología del TDAH. Madrid: Springer; 2012. p. 169-96.

58. Accardo JA, Marcus CL, Leonard MB, Shults J, Meltzer LJ, Elia J. Associations between psychiatric comorbidities and sleep disturbances in children with attention-deficit/ hyperactivity disorder. J Dev Behav Pediatr 2012; 33: 97-105.

59. Crespo-Eguílaz N, Narbona J. Trastorno de aprendizaje procedimental: características neuropsicológicas. Rev Neurol 2009; 49: 409-16.

60. Crespo-Eguílaz N, Narbona J, Magallón S. Disfunción de la coherencia central en niños con trastorno de aprendizaje procedimental. Rev Neurol 2012; 55: 513-9.

\section{Depressive phenomenology at the outset of neuropaediatric diseases}

Introduction. Depressive phenomenology, in its diverse nosological forms, affects 8-10\% of children and adolescents of general population. Most frequently mood disorders have a primary origin, following poligenic multifactorial model. Moreover there is a non negligible proportion of cases in which depressive symptoms accompany neurological illnesses or they even constitute a part of predominant manifestations at the clinical start of neurologic disease, or mark a point of inflexion in its course. The aim of the present article is to review relevant literature dealing with this topic.

Development. A significative higher frequency of depressive phenomenology, not explainable by hazard, can be an early manifestation in children and adolescents with: epileptic syndromes, sleep disorders, chronic recurrent cephalalgias, several neurometabolic diseases, and intracranial tumors. Points of coincidence have been shown in dysequilibrium of brain neurotransmitters (serotonine, noradrenaline, hyperglutamatergic states) which could not be hypothesized as maintaining both neurological and mood conditions.

Conclusions. The suspiction of a neurological disease should be adopted in cases of mood disorder not easily explainable by familial antecedents or clear biographical stressors. The search for a brain disorder by means of a meticulous anamnesis and neurological clinical and ancillary exams makes possible to discover the brain disorder at a very early stage and ameliorate the chances of accurately manage both the neurological and mood disorders. The simultaneous treatment of both dimension of the illness helps to improve the patients' quality of life.

Key words. ADHD. Brain tumour. Depression in children and adolescents. Epilepsy. Primary chronic headache. Sleep disorders. 